ACCEPted to ApJ

Preprint typeset using $\mathrm{LAT}_{\mathrm{E}} \mathrm{X}$ style emulateapj v. 5/2/11

\title{
MMT HYPERVELOCITY STAR SURVEY. II. FIVE NEW UNBOUND STARS
}

\author{
Warren R. Brown, Margaret J. Geller, and Scott J. Kenyon \\ Smithsonian Astrophysical Observatory, 60 Garden St, Cambridge, MA 02138 \\ Accepted to ApJ
}

\begin{abstract}
We present the discovery of five new unbound hypervelocity stars (HVSs) in the outer Milky Way halo. Using a conservative estimate of Galactic escape velocity, our targeted spectroscopic survey has now identified 16 unbound HVSs as well as a comparable number of HVSs ejected on bound trajectories. A Galactic center origin for the HVSs is supported by their unbound velocities, the observed number of unbound stars, their stellar nature, their ejection time distribution, and their Galactic latitude and longitude distribution. Other proposed origins for the unbound HVSs, such as runaway ejections from the disk or dwarf galaxy tidal debris, cannot be reconciled with the observations. An intriguing result is the spatial anisotropy of HVSs on the sky, which possibly reflects an anisotropic potential in the central 10-100 pc region of the Galaxy. Further progress requires measurement of the spatial distribution of HVSs over the southern sky. Our survey also identifies seven B supergiants associated with known star-forming galaxies; the absence of B supergiants elsewhere in the survey implies there are no new star-forming galaxies in our survey footprint to a depth of 1-2 Mpc.

Subject headings: Galaxy: halo - Galaxy: center - Galaxy: kinematics and dynamics — stars: early-type - galaxies: individual (M31, Sextans B)
\end{abstract}

\section{INTRODUCTION}

Unbound radial velocities distinguish HVSs from other main sequence stars in the Galaxy. In Brown et al. (2005) we reported the first HVS: a short-lived $3 M_{\odot}$ star traveling with a Galactic rest frame velocity of $700 \pm 12 \mathrm{~km} \mathrm{~s}^{-1}$, twice the Milky Way's escape velocity at the star's distance of $\simeq 100 \mathrm{kpc}$. The observed motion of the star is comparable to the escape velocity from the surface of the star and is thus difficult to explain with stellar dynamics. The maximum ejection velocity from stellar binary disruption mechanisms Blaauw 1961; Poveda et al. 1967) is limited to $\sim 300 \mathrm{~km} \mathrm{~s}^{-1}$ for $3 M_{\odot}$ stars (Leonard \& Duncan 1988, 1990; Leonard 1991, 1993; Tauris \& Takens 1998; Portegies Zwart 2000; Davies et al. 2002; Gualandris et al. 2005).

There is overwhelming evidence for a $4 \times 10^{6} \mathrm{M}_{\odot}$ massive black hole $(\mathrm{MBH})$ in the Galactic center (Ghez et al. 2008; Gillessen et al. 2009b). The MBH sits in a vast crowd of stars, including short-lived B stars with orbital periods as short as 15 years (Gillessen et al. 2009a) and pericenter velocities as high as $12,000 \mathrm{~km} \mathrm{~s}^{-1}$ (=4\% of the speed of light) (Ghez et al. 2005). Three-body interactions between stars and the $\mathrm{MBH}$ are inevitable in this environment and will naturally result in the ejection of unbound "hypervelocity stars" (Hills 1988). This ejection process also works for a binary $\mathrm{MBH}$ (Yu \& Tremaine 2003).

A MBH in the Galactic center must produce HVSs, and known HVSs fit the MBH ejection picture. The observed unbound velocities, stellar nature, ejection time distribution, and Galactic latitude and longitude distribution of HVSs all support a Galactic center origin. Known HVSs have the spectral types of B stars, the same spectral type as the stars observed orbiting the central MBH. In all cases where photometric variability (Fuentes et al. 2006) or echelle spectroscopy (Przybilla et al. 2008b c; López-Morales \& Bonanos 2008) is available, HVSs are confirmed to be short-lived main sequence B stars. Even the observed number of HVSs is consistent with the theoretically predicted MBH ejection rate (Yu \& Tremaine 2003; Perets et al. 2007) and with the number of "S-stars" presently orbiting the MBH (Perets 2009a; Bromley et al. 2012). In the Hills three-body exchange scenario, S-stars are the former companions of HVSs (Ginsburg \& Loeb 2006), a picture which may (Perets et al. 2009a) or may not (Madigan et al. 2011) be supported by the S-stars' eccentricity distribution. Proper motion measurements promise to more directly test the link between HVSs and the MBH in the Galactic center (e.g., Brown et al. 2010b).

Here, we present the results of our on-going spectroscopic survey on the $6.5 \mathrm{~m}$ MMT telescope to find new HVSs. Our effective survey strategy is to target stars with the colors of $\simeq 3 M_{\odot}$ stars - stars bluer than halo blue horizontal branch (BHB) stars but redder than foreground white dwarfs - that should not exist at faint magnitudes unless they were ejected into the outer halo as HVSs. In previous papers we reported the discovery of 14 unbound HVSs and a comparable population of possibly bound HVSs over a fifth of the sky (Brown et al. 2006a,b, 2007b,c, 2009a b). Here we report 5 new unbound HVSs; the distribution of HVS angular positions on the sky is significantly anisotropic.

In $\S 2$ we describe our HVS survey strategy and spectroscopic observations. In $\S 3$ we discuss the distribution of stars in the survey, and identify the bound and unbound HVSs. In $\S 4$ we discuss the HVSs, and in $\S 5$ we discuss their spatial anisotropy. We conclude in $\S 6$.

\section{DATA}

\subsection{Target Selection Strategy}

We select HVS candidates with the magnitudes and colors of 2.5-4 $M_{\odot}$ stars in the outer halo. 2.5-4 $M_{\odot}$ stars are luminous and allow us to explore a large volume of space. Selecting these stars maximizes our contrast with 


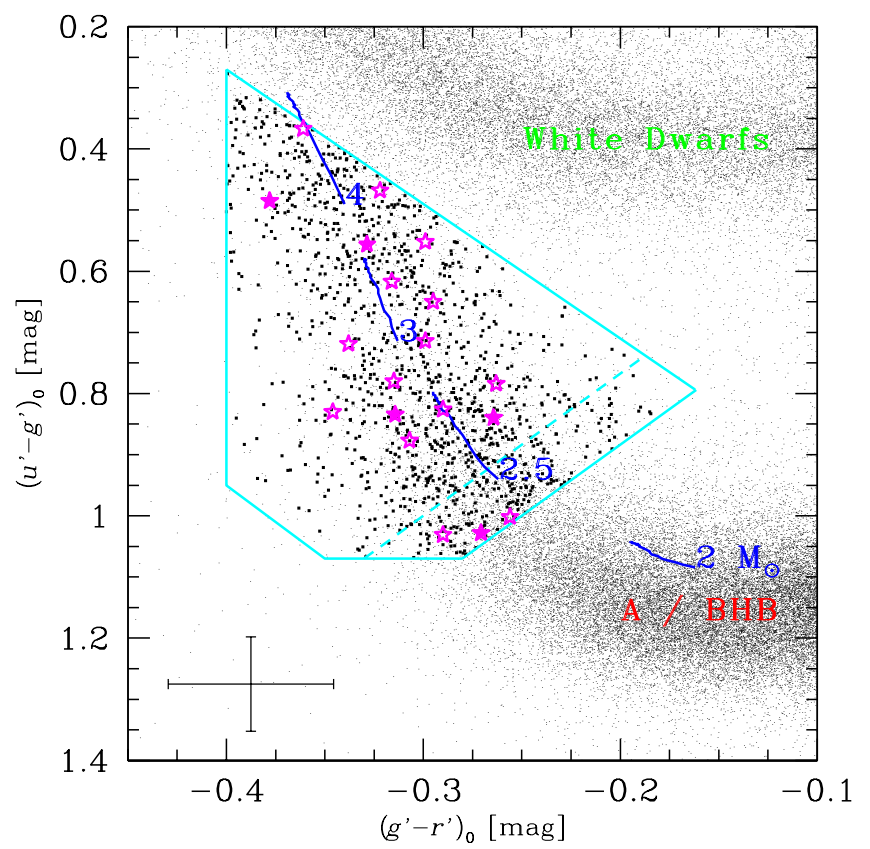

FIG. 1.- Color-color diagram showing the target selection for the unified HVS Survey (solid cyan line) and the observed candidates (black squares). The Survey samples $17<g<20.25$ to the left of the dashed line and $19<g<20.25$ to the right of the dashed line as described in the text. Five new HVSs (solid magenta stars) and the fourteen previous HVSs (open magenta stars) scatter around the Girardi et al. (2004) stellar evolution tracks for 2.5-4 $\mathrm{M}_{\odot}$ main sequence stars (solid blue lines); numbers along the tracks indicate stellar mass. Average SDSS photometric uncertainties for a $g_{0}=19.5$ star are indicated by the errorbars on the lower left. For reference, we plot the underlying distribution of stars in SDSS (black dots) and label the locus of white dwarfs and A-type/BHB stars.

respect to the Milky Way's stellar population, because 2.5-4 $M_{\odot}$ stars are bluer than halo BHB stars and redder than disk white dwarfs. Targeting faint stars maximizes our efficiency for detecting HVSs because the density of normal Milky Way stars is very low in the outer halo. Finally, 2.5-4 $M_{\odot}$ stars have main sequence lifetimes of a few $\times 10^{8}$ yr and thus should not exist at large distances unless they were ejected there.

We select candidates based on de-reddened point spread function photometry from the Sloan Digital Sky Survey (SDSS, Aihara et al. 2011). Each SDSS data release changes its photometric calibration, however, which means that each data release contains candidates unique to that data release plus candidates in common with other data releases. Because we observe candidates taken from each subsequent data release (DR), our HVS Survey is a concatenation of candidates selected from SDSS DR6, DR7, and DR8.

We begin with a cut on reddening $E(B-V)<0.1$ to ensure accurate colors. We exclude the small region of the SDSS between $b<-l / 5+50^{\circ}$ and $b>l / 5-50^{\circ}$ to avoid excessive contamination from Galactic bulge stars. We also impose $-0.5<(r-i)_{0}<0$ to eliminate nonstellar objects such as quasars.

Figure1illustrates our color selection. This color selection unifies the original HVS survey that targets brighter and bluer objects (Brown et al. 2006a, b, 2007b, c) and our more recent HVS survey that targets fainter and red- der objects (Brown et al. 2009a). For reference, we plot in Figure 1 the underlying distribution of stars in SDSS and main sequence tracks for $2 M_{\odot}, 2.5 M_{\odot}, 3 M_{\odot}$, and $4 M_{\odot}$ solar metallicity stars (Girardi et al. 2002, 2004). Our color selection region targets the sequence of $2.5-$ $4 M_{\odot}$ stars bounded by $-0.4<(g-r)_{0}<(-0.43(u-$ $\left.g)_{0}+0.18\right)$ and $\left(2.2(g-r)_{0}+1.1\right)<(u-g)_{0}<1.07$.

Our magnitude selection is $17<g_{0}<20.25$ in the region $(g-r)_{0}<\left(-0.43(u-g)_{0}+0.13\right)$. In the region $(g-r)_{0}>\left(-0.43(u-g)_{0}+0.13\right)$ we restrict ourselves to fainter magnitudes $18.6+10\left[(g-r)_{0}+0.3\right]+\left[(u-g)_{0}-\right.$ $\left.\left(1.2(g-r)_{0}+1.25\right)\right]<g_{0}<20.25$, or approximately $19<g_{0}<20.25$.

Applying these selection criteria to the SDSS DR6, DR7, and DR8 photometric catalogs results in 874, 1097, and 1424 candidates, respectively. After removing objects such as nearby galaxies and bright stars by visual inspection, 1509 unique candidates remain. We previously published spectroscopic identifications and radial velocities for 609 of these candidates (plus a few hundred others that fall outside the present selection criteria) summarized in Brown et al. (2010b). SDSS provides spectroscopy for another 63 candidates. Thus there remain 837 candidates to be observed; we report observations of 497 of these candidates here.

\subsection{Spectroscopic Observations}

We obtained spectroscopy for 497 candidates at the $6.5 \mathrm{~m}$ MMT telescope during observing runs spanning December 2008 to October 2011. All observations were obtained with the Blue Channel spectrograph (Schmidt et al. 1989) using the 832 line $\mathrm{mm}^{-1}$ grating in second order and either a $1^{\prime \prime}$ or $1^{\prime \prime} .25$ slit. These settings provide wavelength coverage $3600 \AA$ to $4500 \AA$ and a spectral resolution of $1-1.2 \AA$. All observations were obtained at the parallactic angle and were paired with comparison lamp exposures.

Our goal was to obtain modest signal-to-noise $(S / N)$ observations adequate for determining radial velocity. At $g=19$ mag we typically used a $390 \mathrm{~s}$ exposure to obtain $(S / N) \simeq 7$ pix $^{-1}$ in the continuum. We processed the spectra in real-time to allow additional observations of interesting candidates. We extracted the spectra using IRAFI in the standard way and measured radial velocities using the cross-correlation package RVSAO (Kurtz \& Mink 1998). The average statistical uncertainty of the radial velocity measurements is \pm 11 $\mathrm{km} \mathrm{s}^{-1}$.

While we made an effort not to observe stars with existing spectroscopy, SDSS re-observed 245 of our stars as part of SEGUE (Yanny et al. 2009). We can use this independent data set to verify our velocity calibration. The mean difference between our heliocentric radial velocities and elodiervfinal from SDSS DR8 is $2 \pm 27 \mathrm{~km} \mathrm{~s}^{-1}$. The dispersion is consistent with the statistical uncertainties; $2 \mathrm{~km} \mathrm{~s}^{-1}$ is our systematic uncertainty.

Two epochs of spectroscopy also allow us to identify objects with variable radial velocity. Although no HVS exhibits a significant change in radial velocity, there are

1 IRAF is distributed by the National Optical Astronomy Observatories, which are operated by the Association of Universities for Research in Astronomy, Inc., under cooperative agreement with the National Science Foundation. 
eight probable white dwarfs that exhibit $>4-\sigma$ changes in velocity between the two epochs. We expect that these objects are compact binaries, and we are pursuing follow-up observations to confirm their velocity variability. To date, follow-up of low mass $<0.25 M_{\odot}$ white dwarfs in the HVS Survey has resulted in the discovery of two dozen merging, double-degenerate binaries with orbital periods as short as 12 minutes (Brown et al. 2010c, 2011a, b, 2012; Kilic et al. 2010, 2011a, b. .c, 2012).

\subsection{Survey Completeness and Spectroscopic Identifications}

With 1169 spectra in hand, the HVS survey is now $90 \%$ complete for DR6- and DR7-selected candidates and $43 \%$ complete for DR8-selected candidates. The remaining candidates are concentrated at faint magnitudes $19.75<$ $g_{0}<20.25$ but are spread relatively evenly over the sky. This distribution of incompleteness is the result of many poor observing runs that prevented observations of the faintest objects in the survey.

Of the 1169 objects: $955(82 \%)$ are normal stars with late-B spectral types; $192(16 \%)$ are white dwarfs; 15 $(1 \%)$ are quasars at $z \sim 2.4$; and $7(1 \%)$ are B supergiant stars. The width and shape of the hydrogen Balmer lines provide a measure of surface gravity over our range of color (temperature). We use line indices described by Brown et al. (2003) to estimate spectral types, and we perform stellar atmosphere model fits for the late B-type objects using an upgraded version of the code described by Allende Prieto et al. (2006). The white dwarfs are studied in a series of papers on low mass white dwarfs that begin with Kilic et al. (2007a,b). The quasars were reported previously (Brown et al. 2009a). We discuss the $\mathrm{B}$ supergiants below, and focus the remainder of this paper on the 955 late B-type stars in the HVS survey.

\subsection{B Supergiants in the HVS Survey}

Our spectra reveal seven B supergiants in the $\simeq 10,000$ $\mathrm{deg}^{2}$ of sky sampled by our survey. All seven B supergiants are associated with known star-forming galaxies in the Local Group. The first two B supergiants belong to the Leo A dwarf. We used those supergiants plus others to make the first stellar velocity dispersion measurement of Leo A (Brown et al. 2007a).

Four new B supergiants belong to M31 and trace its rotation curve. The stars are SDSS J003748.658+395402.57, SDSS J004108.023+401337.08, SDSS J004828.734+423158.58, and SDSS J004855.598+424629.99. The four stars have a mean apparent magnitude of $g_{0}=19.3$ and thus are located at the distance of M31 (Vilardell et al. 2010) for a typical B9 Ib luminosity of $M_{g} \simeq-5.0$. The stars have angular separations of $1^{\circ}-2^{\circ}$ from the center of M31 and locations consistent with the M31 disk. The two northern stars have systemic velocities of $+230 \mathrm{~km}$ $\mathrm{s}^{-1}$ and $+250 \mathrm{~km} \mathrm{~s}^{-1}$ with respect to M31, and the two southern stars have systemic velocities of -126 $\mathrm{km} \mathrm{s}^{-1}$ and $-200 \mathrm{~km} \mathrm{~s}^{-1}$ with respect to M31. The velocities perfectly match the HI rotation curve of M31 (Corbelli et al. 2010); the B supergiants clearly belong to the outer disk of M31.

The final B supergiant, SDSS J095951.180+052124.52, lies within 2.5 of the center of Sextans B. The star's

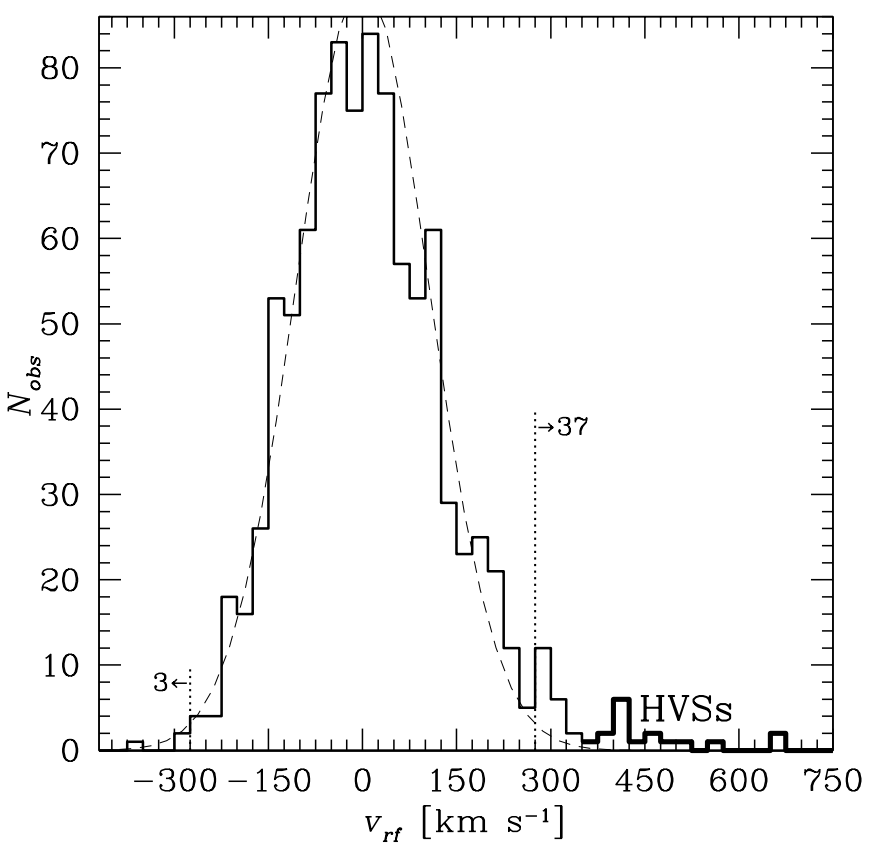

FIG. 2.- Minimum Galactic rest-frame velocity $v_{r f}$ distribution for the 955 late B-type stars in the HVS survey. The best-fit Gaussian (dashed line) has dispersion $107 \mathrm{~km} \mathrm{~s}^{-1}$, excluding the 39 stars with $\left|v_{r f}\right|>275 \mathrm{~km} \mathrm{~s}^{-1}$. The significant absence of $v_{r f}<-275$ $\mathrm{km} \mathrm{s}^{-1}$ stars demonstrates that the positive velocity outliers are short-lived. Stars with $v_{r f}>400 \mathrm{~km} \mathrm{~s}^{-1}$ are unbound.

$295 \pm 4 \mathrm{~km} \mathrm{~s}^{-1}$ heliocentric radial velocity is identical with the velocity of Sextans B (Falco et al. 1999). Thus this B supergiant almost certainly belongs to Sextans $\mathrm{B}$, and its existence is further evidence for on-going star formation in this Local Group dwarf. For a distance modulus of $(m-M)_{0}=25.56 \pm 0.10$ (Sakai et al. 1997) the $g_{0}=19.22$ star must have $M_{g}=-6.3$, consistent with a B9 Ia supergiant.

With absolute magnitudes of $M_{g}=-5$ to -6 , the HVS Survey can detect B9 Ib/a supergiants to a depth of 1-2 Mpc. The only known star-forming galaxy undetected in our survey footprint is Pegasus, a dwarf that is known to have an extremely weak blue plume (Massey et al. 2007). M33 also falls in our footprint and was detected by our color selection, however we excluded M33 stars from the observing list because of obvious extended emission surrounding the stars. The absence of B supergiants elsewhere in the HVS Survey implies that there are no new star-forming galaxies in our survey footprint to a depth of 1-2 Mpc.

\section{RESULTS}

We now discuss the properties of our 955 late B-type stars and identify the unbound HVSs. For reference, Galactic escape velocity at the solar circle is $500-600 \mathrm{~km}$ $\mathrm{s}^{-1}$ (Smith et al. 2007) and at $50 \mathrm{kpc}$ it is $300-400 \mathrm{~km}$ $\mathrm{s}^{-1}$ (Gnedin et al. 2010).

\subsection{Radial Velocity Distribution}

Figure 2 plots the distribution of line-of-sight velocities corrected to the Galactic rest-frame $v_{r f}$ for the 955 late B-type stars in the HVS Survey. We calculate rest frame velocities using the local standard of rest from Schönrich et al. (2010) and a $250 \mathrm{~km} \mathrm{~s}^{-1}$ circular 
rotation velocity (Reid et al. 2009; McMillan \& Binney 2010):

$$
v_{r f}=v_{\text {helio }}+11.1 \cos l \cos b+262.24 \sin l \cos b+7.25 \sin b .
$$

This definition of $v_{r f}$ changes our previous estimates of Galactic rest-frame velocity by up to $30 \mathrm{~km} \mathrm{~s}^{-1}$. We note that $v_{r f}$ represents the radial component of velocity in the Galactic rest frame, and is thus a lower limit to the true velocity of any HVS.

The 915 survey stars with $\left|v_{r f}\right|<275 \mathrm{~km} \mathrm{~s}^{-1}$ have a $0 \pm 4 \mathrm{~km} \mathrm{~s}^{-1}$ mean and a $107 \pm 5 \mathrm{~km} \mathrm{~s}^{-1}$ dispersion, consistent with a stellar halo population. The velocity distribution, however, is not exactly Gaussian (Figure 2). King et al. (2012) show that $10-17 \%$ of the HVS Survey stars may belong to the Sagittarius stream, which explains the excess of stars in the range -50 to $-150 \mathrm{~km}$ $\mathrm{s}^{-1}$ and at $+100 \mathrm{~km} \mathrm{~s}^{-1}$.

Stars with $v_{r f}>+400 \mathrm{~km} \mathrm{~s}^{-1}$ are unbound and probable HVSs. We observe no stars moving towards us with $v_{r f}<-400 \mathrm{~km} \mathrm{~s}^{-1}$, consistent with the picture that stars with $v_{r f}>+400 \mathrm{~km} \mathrm{~s}^{-1}$ are ejected from the Milky Way. The most negative velocity star in our sample has $v_{r f}=-359 \pm 10 \mathrm{~km} \mathrm{~s}^{-1}$, consistent with Kenvon et al. (2008)'s estimate of escape velocity at $50 \mathrm{kpc}$. If the unbound stars are HVSs, then HVS ejection models show that we must also find a comparable number of bound HVSs in our survey (Bromley et al. 2006; Kenyon et al. 2008).

Indeed, we observe a significant excess of stars around $+300 \mathrm{~km} \mathrm{~s}^{-1}$ that are possibly bound HVSs. As an estimate of significance, there is less than a $10^{-5}$ probability of randomly drawing 18 stars with $275<v_{r f}<325 \mathrm{~km}$ $\mathrm{s}^{-1}$ from the tail of a Gaussian distribution with the observed parameters. Thus the excess of stars around +300 $\mathrm{km} \mathrm{s}^{-1}$ are probably not halo stars. Because the stars around $+300 \mathrm{~km} \mathrm{~s}^{-1}$ are bound to the Milky Way, the absence of a comparable number of stars at $-300 \mathrm{~km}$ $\mathrm{s}^{-1}$ demonstrates that the bound stars must have mainsequence lifetimes less than their $\sim 1$ Gyr orbital turnaround times (Brown et al. 2007c; Kollmeier \& Gould 2007; Yu \& Madau 2007). We simply do not see stars falling back onto the Galaxy with similar velocities. Given the observed colors and spectra, we conclude that the stars around $+300 \mathrm{~km} \mathrm{~s}^{-1}$ are main-sequence $\simeq 3 M_{\odot}$ stars ejected into the outer halo.

\subsection{Unbound Stars}

We require distance estimates to determine whether a high velocity star is unbound. The observations demonstrate that high velocity stars are short-lived main sequence stars. We estimate luminosities by comparing observed colors to Girardi et al. (2002, 2004) stellar evolution tracks (see Figure 11) for solar abundance main sequence stars. We use both $(u-g)_{0}$ and $(g-r)_{0}$ colors to estimate luminosity as described in Brown et al. $(2010 \mathrm{~b})$. We estimate that the precision of our luminosities is $\pm 0.23 \mathrm{mag}$ based on propagating color errors through the stellar evolution tracks with a Monte Carlo technique. We then calculate heliocentric distances using de-reddened apparent magnitudes, and convert to Galactocentric distances $R$ assuming that the Sun is $8 \mathrm{kpc}$ from the Galactic center.

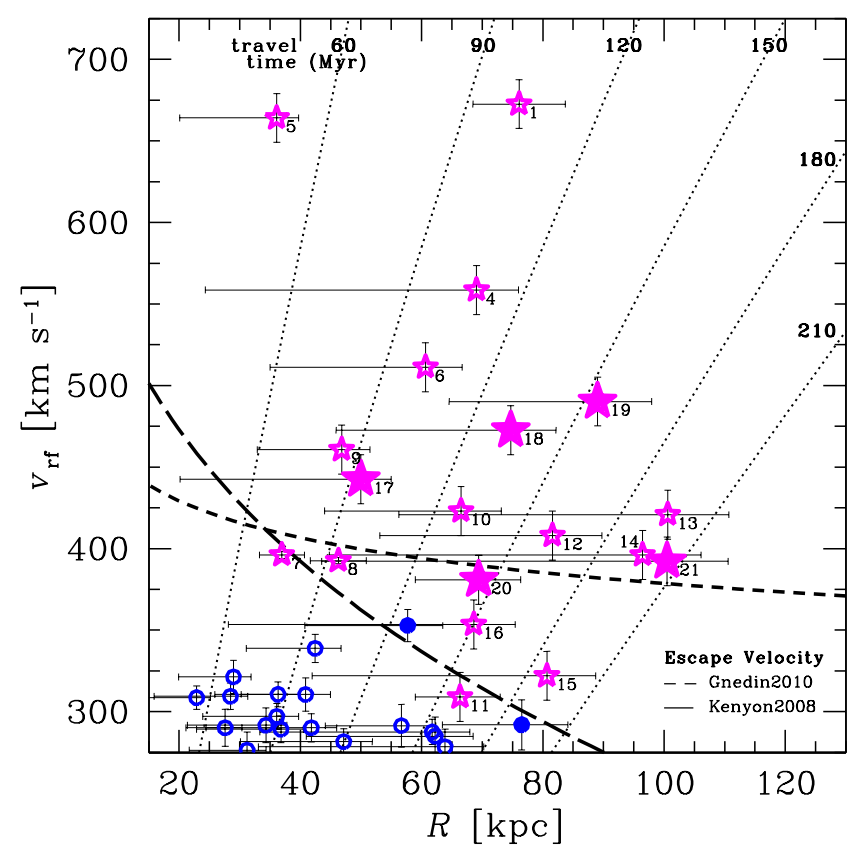

FIG. 3.- Minimum rest-frame velocity vs. Galactocentric distance $R$ for all 37 stars with $v_{r f}>+275 \mathrm{~km} \mathrm{~s}^{-1}$. Distances are estimated using Girardi et al. (2004) main sequence star tracks. Errorbars show the span of physically possible distance given by evolved BHB tracks (Dotter et al. 2008). Five new HVSs (solid magenta stars) have velocities and distances exceeding the Gnedin et al. (2010) escape velocity model (dashed line). Other possible HVSs (solid blue dots) have velocities and distances near the Kenyon et al. (2008) escape velocity model (long dashed line). Previously identified HVSs are marked with open magenta stars. Isochrones of travel time from the Galactic center (dotted lines) are calculated assuming the observed minimum rest frame velocity $v_{r f}$ is the full space motion of the stars.

Systematics are the dominant source of uncertainty in our distance estimates. The Girardi et al. (2002, 2004) absolute magnitudes are $0.24 \pm 0.33$ mag fainter than the Schaller et al. (1992) absolute magnitudes that we used in earlier HVS survey papers. Thus our new luminosity estimates shift known HVSs to smaller distances. HVS7 suffers the biggest change, going from $60 \mathrm{kpc}$ to $37 \mathrm{kpc}$; echelle spectroscopy, however, establishes that HVS7 is a $3.7 M_{\odot}$ Bp star with $M_{V} \simeq-1.05$ (Przybilla et al. $2008 \mathrm{c}$ ) and thus $\simeq 60 \mathrm{kpc}$ distant. This example highlights the uncertainty inherent in stellar evolution tracks. Stellar nature is another contributor to systematic uncertainty. We estimate a physical lower distance limit for our stars by assuming that they are evolved BHB stars. Dotter et al. (2007, 2008) tracks show that hot, metalpoor BHB stars are $1.33 \pm 0.69$ mag fainter than a main sequence star of the same temperature in our survey. We indicate BHB distances with the horizontal error bars in Figure 3 .

Figure 3 plots the resulting distribution of minimum Galactic rest frame velocity $v_{r f}$ versus Galactocentric distance $R$ for the 37 stars with $v_{r f}>+275 \mathrm{~km} \mathrm{~s}^{-1}$. Identifying the unbound stars requires a potential model for the Galaxy. Because the Galactic potential is poorly constrained at large distances, we consider two recent models.

Kenyon et al. (2008) construct a spherically symmetric potential to fit observed Milky Way mass measurements 
TABLE 1

HVS SuRvey Stars With $v_{r f}>+275 \mathrm{KM} \mathrm{S}^{-1}$

\begin{tabular}{|c|c|c|c|c|c|c|c|c|c|}
\hline ID & $\begin{array}{c}v_{\odot} \\
\left(\mathrm{km} \mathrm{s}^{-1}\right)\end{array}$ & $\begin{array}{c}v_{r f} \\
\left(\mathrm{~km} \mathrm{~s}^{-1}\right)\end{array}$ & $\begin{array}{c}g_{0} \\
(\mathrm{mag})\end{array}$ & $\begin{array}{c}M_{g} \\
(\mathrm{mag})\end{array}$ & $\begin{array}{l}R_{G C} \\
(\mathrm{kpc})\end{array}$ & $\begin{array}{c}l \\
(\mathrm{deg})\end{array}$ & $\begin{array}{c}b \\
(\mathrm{deg})\end{array}$ & Catalog & Ref \\
\hline \multicolumn{10}{|c|}{ HVSs } \\
\hline HVS1 & 840 & 673 & 19.69 & +0.41 & 76 & 227.33 & +31.33 & SDSS J090744.99+024506.88 & 1 \\
\hline HVS2 & 708 & 718 & 19.05 & +2.6 & 26 & 175.99 & +47.05 & US708 & 2 \\
\hline HVS3 & 723 & 520 & 16.20 & -2.7 & 62 & 263.04 & -40.91 & HE $0437-5439$ & 3 \\
\hline HVS4 & 611 & 559 & 18.31 & -0.66 & 69 & 194.76 & +42.56 & SDSS J091301.01+305119.83 & 4 \\
\hline HVS5 & 553 & 664 & 17.56 & +0.04 & 36 & 146.23 & +38.70 & SDSS J091759.47+672238.35 & 4 \\
\hline HVS6 & 626 & 511 & 18.97 & +0.10 & 61 & 243.12 & +59.56 & SDSS J110557.45+093439.47 & 5 \\
\hline HVS7 & 529 & 396 & 17.64 & -0.19 & 37 & 263.83 & +57.95 & SDSS J113312.12+010824.87 & 5 \\
\hline HVS8 & 489 & 393 & 17.94 & +0.01 & 46 & 211.70 & +46.33 & SDSS J094214.03+200322.07 & 6 \\
\hline HVS9 & 628 & 461 & 18.64 & +0.34 & 47 & 244.63 & +44.38 & SDSS J102137.08-005234.77 & 6 \\
\hline HVS10 & 478 & 423 & 19.22 & +0.21 & 66 & 249.93 & +75.72 & SDSS J120337.85+180250.35 & 6 \\
\hline HVS12 & 552 & 408 & 19.61 & +0.13 & 82 & 247.11 & +52.46 & SDSS J105009.59+031550.67 & 7 \\
\hline HVS13 & 575 & 421 & 20.02 & +0.09 & 101 & 251.65 & +50.64 & SDSS J105248.30-000133.94 & 7 \\
\hline HVS14 & 532 & 396 & 19.72 & -0.25 & 96 & 241.78 & +53.20 & SDSS J104401.75+061139.02 & 7 \\
\hline HVS17 & 246 & 442 & 17.43 & -0.74 & 50 & 73.52 & +41.16 & SDSS J164156.39+472346.12 & \\
\hline HVS18 & 251 & 473 & 19.30 & +0.05 & 75 & 103.64 & -26.77 & SDSS J232904.94+330011.47 & \\
\hline HVS19 & 597 & 490 & 20.06 & +0.35 & 89 & 256.05 & +63.74 & SDSS J113517.75+080201.49 & \\
\hline HVS20 & 504 & 381 & 19.81 & +0.54 & 69 & 262.56 & +60.39 & SDSS J113637.13+033106.84 & \\
\hline HVS21 & 355 & 392 & 19.73 & -0.23 & 100 & 165.26 & +56.11 & SDSS J103418.25+481134.57 & \\
\hline \multicolumn{10}{|c|}{ Possible HVSs } \\
\hline \multirow[t]{3}{*}{ HVS11 } & 477 & 307 & 19.58 & +0.68 & 64 & 238.77 & +40.63 & SDSS J095906.47+000853.41 & 7 \\
\hline & 222 & 292 & 19.83 & +0.53 & 77 & 155.50 & +49.46 & SDSS J101359.79+563111.65 & 7 \\
\hline & 496 & 353 & 18.86 & +0.28 & 54 & 256.27 & +54.55 & SDSS J111136.44+005856.44 & \\
\hline HVS15 & 463 & 322 & 19.15 & -0.29 & 78 & 266.51 & +55.92 & SDSS J113341.09-012114.25 & 7 \\
\hline HVS16 & 434 & 345 & 19.33 & -0.03 & 74 & 285.86 & +67.38 & SDSS J122523.40+052233.84 & 7 \\
\hline \multicolumn{10}{|c|}{ Possible Bound HVSs } \\
\hline & 150 & 321 & 17.14 & +0.29 & 27 & 115.82 & -40.58 & SDSS J002810.33+215809.66 & \\
\hline & 136 & 311 & 17.77 & +0.03 & 40 & 125.05 & -31.26 & SDSS J005956.06+313439.29 & \\
\hline & 108 & 276 & 17.19 & +0.18 & 30 & 127.53 & -31.42 & SDSS J010948.30+311727.66 & \\
\hline & 361 & 288 & 18.39 & -0.25 & 61 & 196.07 & +23.21 & SDSS J074950.24+243841.16 & 7 \\
\hline & 229 & 297 & 17.28 & +0.06 & 34 & 160.45 & +34.20 & SDSS J081828.07+570922.07 & 7 \\
\hline & 306 & 282 & 18.08 & +0.14 & 45 & 186.30 & +42.16 & SDSS J090710.07+365957.54 & 7 \\
\hline & 504 & 339 & 18.48 & +0.39 & 44 & 247.97 & +46.42 & SDSS J103357.26-011507.35 & \\
\hline & 448 & 285 & 19.23 & +0.14 & 68 & 250.71 & +47.87 & SDSS J104318.29-013502.51 & \\
\hline & 482 & 309 & 17.38 & +0.08 & 30 & 269.75 & +47.30 & SDSS J112255.77-094734.92 & \\
\hline & 140 & 291 & 19.13 & +0.47 & 58 & 130.08 & +40.59 & SDSS J112359.47+751807.73 & \\
\hline & 424 & 290 & 18.13 & -0.07 & 44 & 274.88 & +57.45 & SDSS J115245.91-021116.21 & 7 \\
\hline & 228 & 309 & 17.49 & +0.06 & 31 & 65.34 & +72.37 & SDSS J140432.38+352258.41 & 7 \\
\hline & 284 & 289 & 18.40 & +0.22 & 40 & 357.16 & +63.62 & SDSS J141723.34+101245.74 & 7 \\
\hline & 206 & 278 & 18.88 & -0.12 & 58 & 18.68 & +44.85 & SDSS J154806.92+093423.93 & \\
\hline & 62 & 292 & 17.51 & +0.12 & 29 & 75.71 & +28.06 & SDSS J180050.86+482424.63 & \\
\hline & 133 & 290 & 17.35 & +0.41 & 25 & 85.47 & -51.67 & SDSS J232229.47+043651.45 & \\
\hline
\end{tabular}

RefERences. - (1) Brown et al. (2005); (2) Hirsch et al. (2005); (3) Edelmann et al. (2005); (4) Brown et al.

(2006a); (5) Brown et al. (2006b); (6) Brown et al. (2007c); (7) Brown et al. (2009a)

from $5 \mathrm{pc}$ to $10^{5} \mathrm{pc}$. We estimate escape velocity from this model by dropping a test particle from rest at 250 kpc and calculating its infall velocity. This calculation yields an escape velocity of $360 \mathrm{~km} \mathrm{~s}^{-1}$ at $50 \mathrm{kpc}$ (longdashed line in Figure 3). Gnedin et al. (2010) measure the mass profile of the Milky Way using the velocity dispersion of the (bound) HVS Survey stars. Assuming that the escape velocity is twice the circular velocity, we estimate an escape velocity of $400 \mathrm{~km} \mathrm{~s}^{-1}$ at $50 \mathrm{kpc}$ (dashed line in Figure 3). The escape velocity profiles differ in part because the Gnedin et al. (2010) dark matter halo is $1.6 \times 10^{12} M_{\odot}$ while the Kenvon et al. (2008) dark matter halo is $1 \times 10^{12} M_{\odot}$. The difference in escape velocity profiles illustrates the present uncertainties. Because a larger halo mass is preferred by recent observations (e.g., Przybilla et al. 2010), we use the Gnedin et al. (2010) model to identify new unbound stars.
We identify five new unbound HVSs with radial velocities and distances equaling or exceeding the Gnedin et al. (2010) escape velocity model, and one new possible HVS exceeding the Kenyon et al. (2008) escape velocity model. Previously we identified 14 unbound HVSs on the basis of the Kenvon et al. (2008) model, however our new $v_{r f}$ and distance estimates place HVS11 slightly below the threshold of the Kenyon et al. (2008) model. Another two stars, HVS15 and HVS16, are above the threshold of the Kenyon et al. (2008) model but well below the threshold of the Gnedin et al. (2010) model. Therefore we re-classify these three objects as "possible HVSs." HVS7 and HVS8, on the other hand, are demonstrated $\simeq 4 M_{\odot}$ main sequence B stars at $R \simeq 50 \mathrm{kpc}$ (López-Morales \& Bonanos 2008; Przybilla et al. 2008c) and thus are almost certainly HVSs.

Table 1 summarizes the properties of all 37 HVS Survey stars with $v_{r f}>+275 \mathrm{~km} \mathrm{~s}^{-1}$. Columns include 
HVS number, heliocentric radial velocity $v_{\odot}$, minimum Galactic rest-frame velocity $v_{r f}$ (not a full space velocity), de-reddened SDSS $g$-band magnitude, absolute magnitude $M_{g}$ from Girardi et al. (2002, 2004) main sequence tracks, Galactocentric distance $R$, Galactic coordinates $(l, b)$, and catalog identification. There are 16 unbound HVSs, 5 possible HVSs, and 16 possibly bound HVSs.

The velocity and distance estimates allow us to estimate ejection times. The dotted lines in Figure 3 indicate travel times from the Galactic center under the assumption that $v_{r f}$ is the 3 -dimensional velocity of our stars, a conservative assumption even though the orbits should be nearly radial (Gnedin et al. 2005). We find that HVSs are not clustered around a common ejection time, but have a range of ejection times spread continuously between 60 and $210 \mathrm{Myr}$. In other words, the observations do not support a large burst of HVSs that one might expect for binary MBH in-spiral or dwarf galaxy tidal disruption models. Rather, the observations support an on-going ejection process, consistent with the Hills three-body ejection model.

\section{FIVE NEW HYPERVELOCITY STARS}

We discuss the details of the five new unbound HVSs discovered in this portion of the survey. All of the HVSs except for HVS17 have multiple epochs of observation. None of the HVSs exhibit significant radial velocity variations, which indicates that the HVSs are unlikely to be compact binaries.

SDSS J164156.391+472346.12, hereafter HVS17, has a $+246 \pm 9 \mathrm{~km} \mathrm{~s}^{-1}$ heliocentric radial velocity and a minimum velocity of $v_{r f}=+443$ in the Galactic frame. HVS17 is the hottest HVS in our survey with a B6 spectral type. HVS17 is also the brightest HVS in our survey, $g=17.499 \pm 0.015 \mathrm{mag}$, opening the possibility of follow-up echelle spectroscopy. A solar metallicity $4 M_{\odot}$ star with the temperature of HVS17 has $M_{g}=-0.74$ (Girardi et al. 2002, 2004) and a distance of $R=50 \mathrm{kpc}$.

SDSS J232904.947+330011.47, hereafter HVS18, has $\mathrm{a}+251 \pm 10 \mathrm{~km} \mathrm{~s}^{-1}$ heliocentric radial velocity and a minimum velocity of $v_{r f}=+473 \mathrm{~km} \mathrm{~s}^{-1}$ in the Galactic frame. At $b=-26.8^{\circ}$, HVS18 is the lowest latitude HVS in our survey. HVS18 is also our first HVS discovery in the southern Galactic hemisphere. Unlike HVS3, the $9 M_{\odot}$ HVS near the LMC (Edelmann et al. 2005; Przybilla et al. 2008b), HVS18 is $17^{\circ}$ from M31. Any association with M31 or its satellites is impossible. A solar metallicity $3 M_{\odot}$ star with the temperature of HVS18 has $M_{g}=0.05$, thus HVS18 has a distance of $R=75$ $\mathrm{kpc}$ from the Milky Way and a distance of $700 \mathrm{kpc}$ from M31.

SDSS J113517.759+080201.49, hereafter HVS19, has a $+597 \pm 15 \mathrm{~km} \mathrm{~s}^{-1}$ heliocentric radial velocity and a minimum velocity of $v_{r f}=+490 \mathrm{~km} \mathrm{~s}^{-1}$ in the Galactic frame. HVS19 is one of the cooler HVSs in our survey with an A0 spectral type. A solar metallicity $\simeq 2.5 M_{\odot}$ star with the temperature of HVS19 has $M_{g}=0.35$ which locates it at $R=90 \mathrm{kpc}$. Like many of the other HVSs, HVS19 is in the constellation of Leo.

SDSS J113637.135+033106.84, hereafter HVS20, has a $504 \pm 12 \mathrm{~km} \mathrm{~s}^{-1}$ heliocentric radial velocity and a minimum velocity of $v_{r f}=+381 \mathrm{~km} \mathrm{~s}^{-1}$ in the Galactic frame. HVS20 sits on the escape velocity threshold of
Gnedin et al. (2010) but is well above the threshold of Kenyon et al. (2008). HVS20 is similar to HVS19, only 4.5 away; it is yet another HVS in the constellation Leo. HVS20 is also one of the coolest HVSs in our survey with an A1 spectral type. With an estimated luminosity of $M_{g}=0.54$, HVS20 sits at $R=80 \mathrm{kpc}$ and so shares a similar ejection time as HVS19. The other eight HVSs around Leo have ejection times that differ by up to 125 Myr, however, implying that the HVSs in the direction of Leo do not all come from a single ejection event.

SDSS J103418.254+481134.57, hereafter HVS21, has a $+355 \pm 10 \mathrm{~km} \mathrm{~s}^{-1}$ heliocentric radial velocity and a minimum velocity of $v_{r f}=+392 \mathrm{~km} \mathrm{~s}^{-1}$ in the Galactic frame. HVS21 has a B7 spectral type and, for a luminosity of $M_{g}=-0.23$, is located at $R=100 \mathrm{kpc}$. Like HVS20, HVS21 sits on escape velocity threshold of Gnedin et al. (2010) but is well above the threshold of Kenvon et al. (2008). HVS21 is in Ursa Major about $11^{\circ}$ from HVS2 (Hirsch et al. 2005).

\section{SPATIAL ANISOTROPY}

The spatial distribution of HVSs on the sky is intriguing because it is almost certainly linked to their origin. In principle, the MBH in the Galactic center can eject HVSs in all directions. Brown et al. (2009b) demonstrate that unbound HVSs have a significantly anisotropic distribution on the sky. Lower velocity bound HVSs have a more isotropic distribution. The new HVSs reported here support these trends.

Figure 4plots the angular positions of all of the survey stars. The overall distribution of positions reflects the SDSS imaging footprint. HVSs are marked with solid magenta stars and possible HVSs are marked with solid blue dots. The stars have a similar distribution and so we consider the combined set of HVSs in an attempt to mitigate small number statistics. Because of the complex SDSS footprint and because of the HVS Survey incompleteness, we quantify the spatial anisotropy by comparing the distribution of HVSs against the distribution of observed stars from which they are drawn. Although this comparison is not perfect - the overdensity of stars arcing to the right of the North Galactic pole in Figure 4 is the Sgr stream - this approach provides a fair comparison of positions and angular separations in the HVS survey.

Figure 5 plots the cumulative Galactic longitude and latitude distributions of our survey stars and HVSs. Kolmogorov-Smirnov (K-S) tests find 0.013 and 0.415 likelihoods that the combined set of HVSs are drawn from the same longitude and latitude distributions, respectively, as the survey stars. The longitude and latitude tests are not completely independent because of the complex boundaries of our survey region. The KS tests establish, however, that the HVS spatial anisotropy is primarily in Galactic longitude and not in Galactic latitude.

Next we consider the distribution of angular separations. Seven of the HVSs (33\%) are in the constellation Leo, thus we expect that the HVSs have an excess of small angular separations and an absence of large angular separations compared to the survey stars. Calculating the angular separations for all unique pairs of stars, a K-S test finds a $3 \times 10^{-18}$ likelihood that the HVSs are drawn from the same distribution of angular separations 

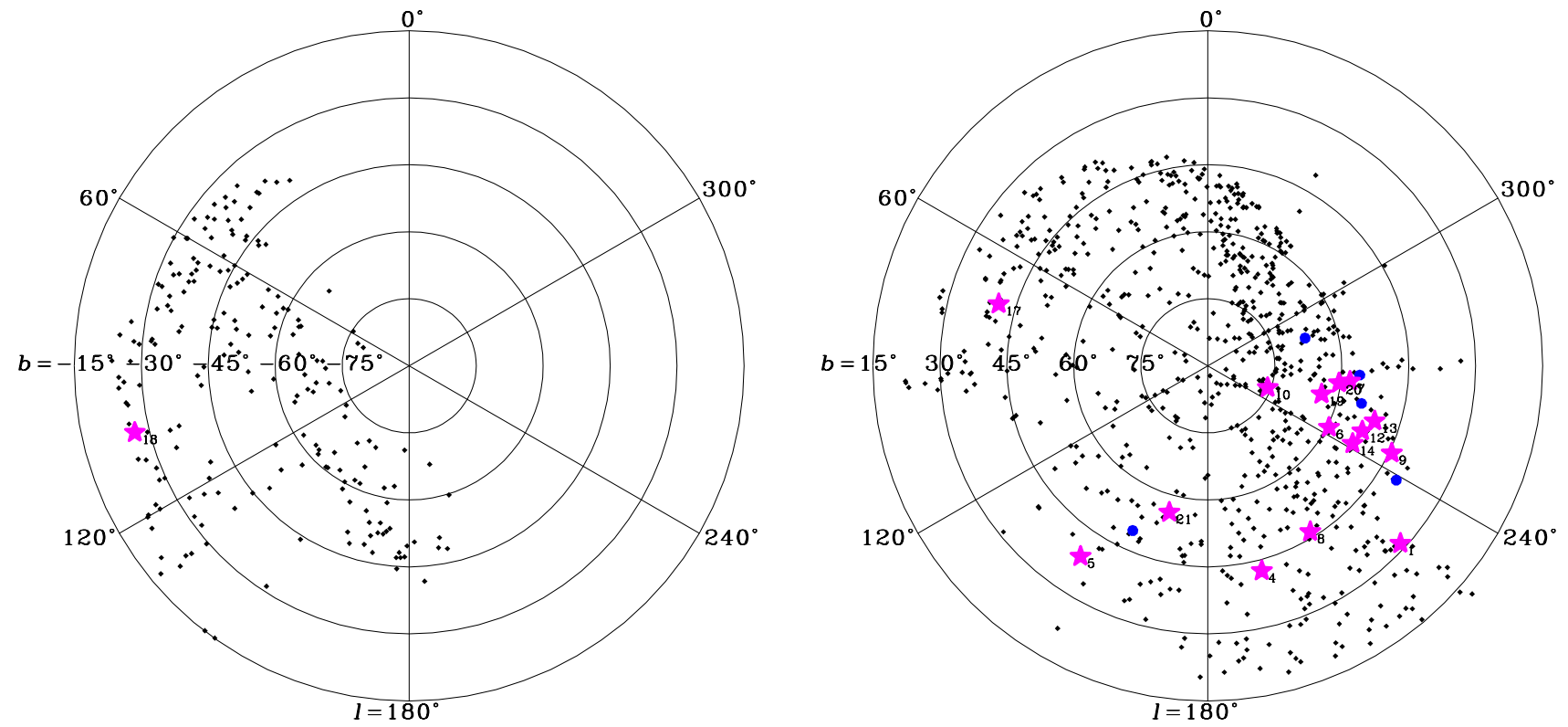

FIG. 4.- Polar projections, in Galactic coordinates, showing the 16 unbound HVSs (magenta stars), the 5 possible HVSs (blue circles) and 934 other stars (black dots) in the HVS survey over the southern (left) and northern (right) Galactic caps.
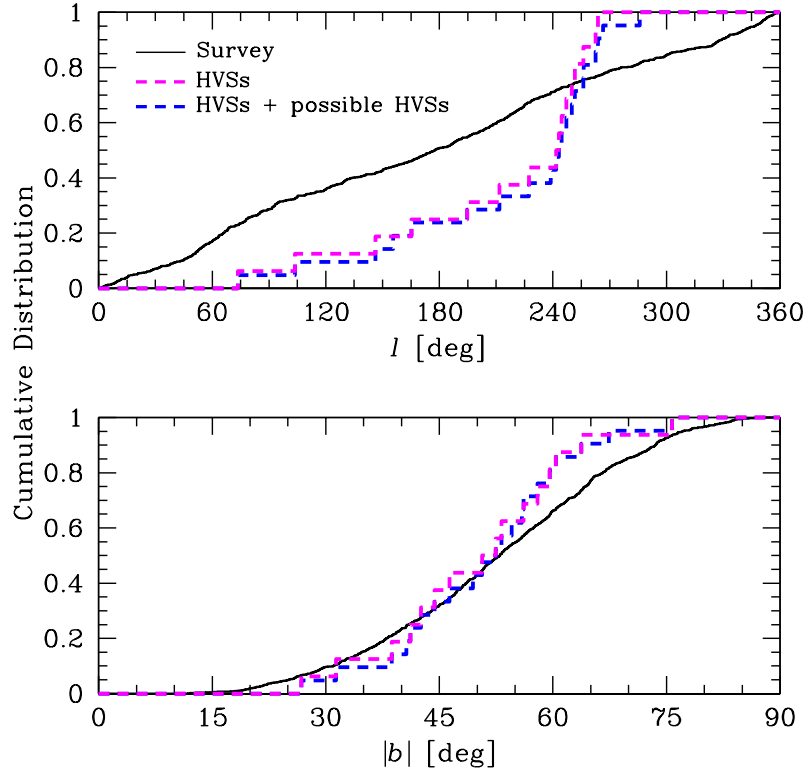

Fig. 5.- Cumulative distributions of Galactic $l$ and $b$ of the 16 unbound HVSs (dashed magenta line), 21 HVSs and possible HVSs (dashed blue line), and the other stars (solid line) in the HVS survey.

as the survey stars. We establish the significance of this likelihood by taking 10,000 random draws of 21 unique stars from the survey: a likelihood below $3 \times 10^{-18}$ occurs at random $0.3 \%$ of the time in our survey. The actual significance of the anisotropy depends on the method of calculation; our approach indicates a formal significance of $3-\sigma$.

A number of models propose to explain the HVS spatial anisotropy. Abadi et al. (2009) propose a tidal debris origin for HVSs (see also Piffl et al. 2011). However, this model is difficult to reconcile with the observations be- cause 1) known HVSs have ejection times that differ by up to $150 \mathrm{Myr}, 2$ ) no other unbound tidal debris is observed in the same region of sky (Kollmeier et al. 2009, 2010), and 3) no dwarf galaxy in the Local Group has comparable velocity. An intermediate mass black hole in-spiral event in the Galactic center may produce a ring of HVSs around the sky (Gualandris et al. 2005; Levin 2006; Sesana et al. 2006; Baumgardt et al. 2006) but this model is also at variance with the observations. Theoretically predicted black hole in-spirals occur on timescales 10-100 times shorter than the observed span of HVS ejection times, thus multiple black hole in-spiral events with common alignment are necessary to account for the observed spatial distribution.

Runaway stars, stars born in the disk and ejected by binary disruptions, are another possible source of apparent HVSs (e.g. Gvaramadze et al. 2009). Surveys of stars near the Galactic disk identify unbound stars ejected from the disk (Heber et al. 2008; Przvbilla et al. 2008a; Tillich et al. 2009; Irrgang et al. 2010) as expected in runaway star mechanisms (Bromley et al. 2009; Gvaramadze 2009). Stars ejected from a rotating disk have a distinct spatial distribution: the fastest stars are those ejected in the direction of Galactic rotation, thus $\simeq 90 \%$ of $>400 \mathrm{~km} \mathrm{~s}^{-1}$ runaways should be located $|b|<45^{\circ}$ (Bromlev et al. 2009). Moreover, $>400 \mathrm{~km}$ $\mathrm{s}^{-1}$ runaways at $R=50 \mathrm{kpc}$ should be found over all Galactic longitudes. Thus the expected distribution of runaway latitudes and longitudes is inconsistent with the observed distribution of HVSs.

The ejection rate of disk runaways is also inconsistent with the observed number of HVSs. The physical size of stars places a speed limit on stellar binary disruption mechanisms: a $3 M_{\odot}$ runaway with $v_{r f}>+400 \mathrm{~km} \mathrm{~s}^{-1}$ at $R=50 \mathrm{kpc}$ requires the disruption of a compact binary containing a massive star within its lifetime while avoiding a merger. An optimistic ejection rate for $3 M_{\odot}$ 
runaways with $>400 \mathrm{~km} \mathrm{~s}^{-1}$ at $R=50 \mathrm{kpc}$ is about 100 to 100,000 times smaller than the expected rate of HVSs ejected by the MBH (Brown et al. 2009a; Perets \& Subr 2012). Given that the HVSs are not clustered at low latitudes in our survey, we conclude that runaways are unlikely contaminants to the HVS sample.

The anisotropic distribution of stars in the Galactic center provides another model for explaining the anisotropy of HVSs. If the central $\mathrm{MBH}$ is the origin of HVSs, and the stars orbiting near the MBH are concentrated in one or two disks (Levin \& Beloborodov 2003; Lu et al. 2009; Bartko et al. 2009), then stars probably interact with the $\mathrm{MBH}$ in preferred planes. Lu et al. (2010) and Zhang et al. (2010) demonstrate that the observed distribution of HVSs is consistent with two thin disk planes aligned with the present stellar disks in the Galactic center. Observed HVSs were ejected up to 200 Myr ago, however. Although the Galactic center clearly contains anisotropic structure - including young stellar clusters like the Arches and Quintuplet clusters and gaseous disks, arms, and bars that may form young stars - there is no model that explains how Galactic center structure maintains a fixed orientation for $200 \mathrm{Myr}$.

A final model is an anisotropic Galactic potential. HVSs are unique test particles because they start in the Galactic center and travel to infinity. The gravitational potential maps initial HVS ejection velocities to the velocities that we observe in the outer halo today. Because many HVSs are marginally unbound, a non-spherical potential can naturally explain why HVSs are found in preferred directions on the sky: stars ejected along the long axis of the potential would have the fastest observed velocities; stars ejected along the minor axis of the potential are decelerated more and so fewer will be observed as HVSs. Kenyon et al. (2008) show that the important quantity is the scale length of potential in the central 10$100 \mathrm{pc}$, the region where HVSs experience the most deceleration. The anisotropic potential model can be tested with the all-sky distribution of HVSs. If there is rotation around the long axis of the potential, stars ejected along the long axis should be located in two clumps on opposite points in the sky. If there is rotation around the short axis, stars ejected along the long axis should appear in ring of HVSs around the sky. There are currently no good constraints on the shape and orientation of the Galactic potential, but perhaps the HVSs will reveal it.

\section{CONCLUSIONS}

We report new results from our targeted HVS Survey, a spectroscopic survey of stars with $\simeq 3 M_{\odot}$ colors that should not exist at faint magnitudes unless they were ejected into the outer halo as HVSs. Our survey is $90 \%$ complete for SDSS DR6- and DR7-selected candidates and $43 \%$ complete for DR8-selected candidates. Spectroscopy reveals that most of the candidates are normal late B-type stars in the halo; other objects include low mass white dwarf binaries and B supergiants in known star-forming galaxies. The absence of B supergiants elsewhere in the HVS Survey implies that there are no new star-forming galaxies to a depth of 1-2 Mpc within our survey footprint.

The velocity distribution of the 955 late B-type stars in the survey is remarkable: 37 stars have $v_{r f}>+275 \mathrm{~km}$ $\mathrm{s}^{-1}$ and 3 stars have $v_{r f}<-275 \mathrm{~km} \mathrm{~s}^{-1}$. The observa- tional signature of a HVS is its unbound velocity, which we determine by comparing observed radial velocities and distances to Galactic potential models. There are 5 new unbound HVSs on the basis of the Gnedin et al. (2010) escape velocity model. The HVS Survey thus identifies 16 unbound HVSs, 5 possible HVSs, and 16 possibly bound HVSs.

A Galactic center origin for the HVSs is supported by their unbound velocities, the observed number of unbound stars, their stellar nature, their ejection time distribution, and their Galactic latitude and longitude distribution. Other proposed origins for the unbound HVSs, including runaway ejections from the disk or dwarf galaxy tidal debris, cannot easily be reconciled with the observations. Although not all unbound stars are necessarily HVSs, the MBH ejection origin provides the best explanation for the observed $\simeq 3 M_{\odot}$ unbound HVSs in our survey.

HVSs are important because their properties are tied to the nature and environment of the $\mathrm{MBH}$ that ejects them (Portegies Zwart et al. 2006; Merritt 2006; Demarque \& Virani 2007; Ginsburg \& Loeb 2007; Gualandris \& Portegies Zwart 2007; Hansen 2007; Lu et al. 2007; Sesana et al. 2007a,b, 2008, 2009; O'Leary \& Loeb 2008; Perets \& Alexander 2008; Perets 2009a,b; Sherwin et al. 2008; Svensson et al. 2008; Löckmann \& Baumgardt 2008; Löckmann et al. 2008, 2009; Chen et al. 2009; Gualandris \& Merritt 2009; Hopman 2009; Madigan et al. 2009; Sari et al. 2010; Antonini et al. 2010, 2011; Baruteau et al. 2011; Ginsburg \& Perets 2012). HVS are also important probes of the dark matter potential through which they move (Gnedin et al. 2005; Yu \& Madau 2007; Wu et al. 2008; Kenvon et al. 2008; Perets et al. 2009b).

An intriguing result of the HVS Survey is the spatial anisotropy of unbound HVSs on the sky. There is as yet no compelling explanation for the anisotropy. We speculate that an anisotropic central potential may explain the observations. In the future, measuring the full space trajectories of the HVSs promises to elucidate the origin of HVSs (Brown et al. 2010a) and better constrain the shape and orientation of the Galactic potential. Further progress requires measuring the spatial distribution of HVSs over the entire sky. We look forward to using the Skymapper Survey (Keller et al. 2007) to identify HVS candidates in coming years.

We thank M. Alegria, A. Milone, and J. McAfee for their assistance with observations obtained at the MMT Observatory, a joint facility of the Smithsonian Institution and the University of Arizona. This project makes use of data products from the Sloan Digital Sky Survey, which is managed by the Astrophysical Research Consortium for the Participating Institutions. This research makes use of NASA's Astrophysics Data System Bibliographic Services. This work was supported by the Smithsonian Institution.

Facilities: MMT (Blue Channel Spectrograph) 


\section{REFERENCES}

Abadi, M. G., Navarro, J. F., \& Steinmetz, M. 2009, ApJ, 691, L63

Aihara, H., Allende Prieto, C., An, D., et al. 2011, ApJS, 193, 29

Allende Prieto, C., Beers, T. C., Wilhelm, R., et al. 2006, ApJ, 636,804

Antonini, F., Faber, J., Gualandris, A., \& Merritt, D. 2010, ApJ, 713,90

Antonini, F., Lombardi, Jr., J. C., \& Merritt, D. 2011, ApJ, 731, 128

Bartko, H., Martins, F., Fritz, T. K., et al. 2009, ApJ, 697, 1741

Baruteau, C., Cuadra, J., \& Lin, D. N. C. 2011, ApJ, 726, 28

Baumgardt, H., Gualandris, A., \& Portegies Zwart, S. 2006, MNRAS, 372, 174

Blaauw, A. 1961, Bull. Astron. Inst. Netherlands, 15, 265

Bromley, B. C., Brown, W. R., Geller, M. J., \& Kenyon, S. J. 2009, ApJ, 706, 925

Bromley, B. C., Kenyon, S. J., Geller, M. J., \& Brown, W. R. 2012, ApJ, submitted

Bromley, B. C., Kenyon, S. J., Geller, M. J., et al. 2006, ApJ, 653, 1194

Brown, W. R., Allende Prieto, C., Beers, T. C., Wilhelm, R., Geller, M. J., Kenyon, S. J., \& Kurtz, M. J. 2003, AJ, 126, 1362

Brown, W. R., Anderson, J., Gnedin, O. Y., et al. 2010a, ApJ, 719, L23

Brown, W. R., Geller, M. J., \& Kenyon, S. J. 2009a, ApJ, 690, 1639

Brown, W. R., Geller, M. J., Kenyon, S. J., \& Bromley, B. C. 2009b, ApJ, 690, L69

Brown, W. R., Geller, M. J., Kenyon, S. J., \& Diaferio, A. 2010b, AJ, 139, 59

Brown, W. R., Geller, M. J., Kenyon, S. J., \& Kurtz, M. J. 2005, ApJ, 622, L33

-. 2006a, ApJ, 640, L35

-. 2006b, ApJ, 647, 303

-. 2007a, ApJ, 666, 231

Brown, W. R., Geller, M. J., Kenyon, S. J., Kurtz, M. J., \& Bromley, B. C. 2007b, ApJ, 660, 311

-. 2007c, ApJ, 671, 1708

Brown, W. R., Kilic, M., Allende Prieto, C., \& Kenyon, S. J. 2010c, ApJ, 723, 1072

-. 2011a, MNRAS, 411, L31

-. 2012, ApJ, 744, 142

Brown, W. R., Kilic, M., Hermes, J. J., et al. 2011b, ApJ, 737 L23

Chen, X., Madau, P., Sesana, A., \& Liu, F. K. 2009, ApJ, 697, L149

Corbelli, E., Lorenzoni, S., Walterbos, R., Braun, R., \& Thilker, D. 2010, A\&A, 511, A89

Davies, M. B., King, A., \& Ritter, H. 2002, MNRAS, 333, 463

Demarque, P. \& Virani, S. 2007, A\&A, 461, 651

Dotter, A., Chaboyer, B., Jevremović, D., et al. 2007, AJ, 134, 376

Dotter, A., Chaboyer, B., Jevremović, D., et al. 2008, ApJS, 178, 89

Edelmann, H., Napiwotzki, R., Heber, U., Christlieb, N., \& Reimers, D. 2005, ApJ, 634, L181

Falco, E. E., Kurtz, M. J., Geller, M. J., et al. 1999, PASP, 111, 438

Fuentes, C. I., Stanek, K. Z., Gaudi, B. S., et al. 2006, ApJ, 636, L37

Ghez, A. M., Salim, S., Hornstein, S. D., et al. 2005, ApJ, 620, 744

Ghez, A. M., Salim, S., Weinberg, N. N., et al. 2008, ApJ, 689, 1044

Gillessen, S., Eisenhauer, F., Fritz, T. K., et al. 2009a, ApJ, 707, L114

Gillessen, S., Eisenhauer, F., Trippe, S., et al. 2009b, ApJ, 692, 1075

Ginsburg, I. \& Loeb, A. 2006, MNRAS, 368, 221

-. 2007, MNRAS, 376, 492

Ginsburg, I. \& Perets, H. B. 2012, MNRAS, submitted

Girardi, L., Bertelli, G., Bressan, A., et al. 2002, A\&A, 391, 195

Girardi, L., Grebel, E. K., Odenkirchen, M., \& Chiosi, C. 2004, A\&A, 422, 205

Gnedin, O. Y., Brown, W. R., Geller, M. J., \& Kenyon, S. J. 2010, ApJ, 720, L108
Gnedin, O. Y., Gould, A., Miralda-Escudé, J., \& Zentner, A. R. 2005, ApJ, 634, 344

Gualandris, A. \& Merritt, D. 2009, ApJ, 705, 361

Gualandris, A. \& Portegies Zwart, S. 2007, MNRAS, 376, L29

Gualandris, A., Portegies Zwart, S. P., \& Sipior, M. S. 2005, MNRAS, 363, 223

Gvaramadze, V. V. 2009, MNRAS, 395, L85

Gvaramadze, V. V., Gualandris, A., \& Portegies Zwart, S. 2009, MNRAS, 396, 570

Hansen, B. M. S. 2007, ApJ, 671, L133

Heber, U., Edelmann, H., Napiwotzki, R., Altmann, M., \& Scholz, R.-D. 2008, A\&A, 483, L21

Hills, J. G. 1988, Nature, 331, 687

Hirsch, H. A., Heber, U., O’Toole, S. J., \& Bresolin, F. 2005, A\&A, 444, L61

Hopman, C. 2009, ApJ, 700, 1933

Irrgang, A., Przybilla, N., Heber, U., Fernanda Nieva, M., \& Schuh, S. 2010, ApJ, 711, 138

Keller, S. C., Schmidt, B. P., Bessell, M. S., et al. 2007, PASA, 24,1

Kenyon, S. J., Bromley, B. C., Geller, M. J., \& Brown, W. R. 2008, ApJ, 680, 312

Kilic, M., Allende Prieto, C., Brown, W. R., \& Koester, D. 2007a, ApJ, 660, 1451

Kilic, M., Brown, W. R., Allende Prieto, C., et al. 2011a, ApJ, 727,3

Kilic, M., Brown, W. R., Allende Prieto, C., \& Kenyon, S. J. 2012, ApJ, submitted

Kilic, M., Brown, W. R., Allende Prieto, C., Kenyon, S. J., \& Panei, J. A. 2010, ApJ, 716, 122

Kilic, M., Brown, W. R., Allende Prieto, C., Pinsonneault, M., \& Kenyon, S. 2007b, ApJ, 664, 1088

Kilic, M., Brown, W. R., Hermes, J. J., et al. 2011b, MNRAS, 418, L157

Kilic, M., Brown, W. R., Kenyon, S. J., et al. 2011c, MNRAS, 413, L101

King, III, C., Brown, W. R., Geller, M. J., \& Kenyon, S. J. 2012, ApJ, accepted

Kollmeier, J. A. \& Gould, A. 2007, ApJ, 664, 343

Kollmeier, J. A., Gould, A., Knapp, G., \& Beers, T. C. 2009 , ApJ, 697, 1543

Kollmeier, J. A., Gould, A., Rockosi, C., et al. 2010, ApJ, 723, 812

Kurtz, M. J. \& Mink, D. J. 1998, PASP, 110, 934

Leonard, P. J. T. 1991, AJ, 101, 562

Leonard, P. J. T. 1993, in ASP Conf. Ser. 45, Luminous High-Latitude Stars, ed. D. Sasselov, 360

Leonard, P. J. T. \& Duncan, M. J. 1988, AJ, 96, 222

-. 1990, AJ, 99, 608

Levin, Y. 2006, ApJ, 653, 1203

Levin, Y. \& Beloborodov, A. M. 2003, ApJ, 590, L33

Löckmann, U. \& Baumgardt, H. 2008, MNRAS, 384, 323

Löckmann, U., Baumgardt, H., \& Kroupa, P. 2008, ApJ, 683, L151

—. 2009, MNRAS, 398, 429

López-Morales, M. \& Bonanos, A. Z. 2008, ApJ, 685, L47

Lu, J. R., Ghez, A. M., Hornstein, S. D., et al. 2009, ApJ, 690, 1463

Lu, Y., Yu, Q., \& Lin, D. N. C. 2007, ApJ, 666, L89

Lu, Y., Zhang, F., \& Yu, Q. 2010, ApJ, 709, 1356

Madigan, A.-M., Hopman, C., \& Levin, Y. 2011, ApJ, 738, 99

Madigan, A.-M., Levin, Y., \& Hopman, C. 2009, ApJ, 697, L44

Massey, P., Olsen, K. A. G., Hodge, P. W., Jacoby, G. H., McNeill, R. T., Smith, R. C., \& Strong, S. B. 2007, AJ, 133 2393

McMillan, P. J. \& Binney, J. J. 2010, MNRAS, 402, 934

Merritt, D. 2006, ApJ, 648, 976

O'Leary, R. M. \& Loeb, A. 2008, MNRAS, 383, 86

-. 2009a, ApJ, 690, 795

- 2009b, ApJ, 698, 1330

Perets, H. B. \& Alexander, T. 2008, ApJ, 677, 146

Perets, H. B., Gualandris, A., Merritt, D., \& Alexander, T. 2009a, ApJ, 702, 884

Perets, H. B., Hopman, C., \& Alexander, T. 2007, ApJ, 656, 709

Perets, H. B., \& Subr, L. 2012, ApJ, submitted

Perets, H. B., Wu, X., Zhao, H. S., et al. 2009b, ApJ, 697, 2096 
TABLE 2

Late B-type Halo Stars in the HVS Survey

\begin{tabular}{cccrrrc}
\hline \hline $\begin{array}{c}\text { R.A. } \\
(\mathrm{hr})\end{array}$ & $\begin{array}{c}\text { Decl. } \\
(\mathrm{deg})\end{array}$ & $\begin{array}{c}g \\
(\mathrm{mag})\end{array}$ & \multicolumn{1}{c}{$\begin{array}{c}v_{\odot} \\
\left(\mathrm{km} \mathrm{s}^{-1}\right)\end{array}$} & $\begin{array}{c}v_{r f} \\
\left(\mathrm{~km} \mathrm{~s}^{-1}\right)\end{array}$ & $\begin{array}{c}l \\
(\mathrm{deg})\end{array}$ & \multicolumn{1}{c}{$\begin{array}{c}b \\
(\mathrm{deg})\end{array}$} \\
\hline $0: 01: 31.115$ & $+26: 46: 55.06$ & $19.671 \pm 0.021$ & $-374.4 \pm 11.8$ & -178.3 & 109.35 & -34.77 \\
$0: 02: 33.817$ & $-09: 57: 06.85$ & $18.578 \pm 0.021$ & $-87.7 \pm 7.7$ & -1.8 & 86.76 & -69.32 \\
$0: 05: 28.141$ & $-11: 00: 10.07$ & $19.271 \pm 0.042$ & $-115.9 \pm 9.2$ & -35.5 & 86.89 & -70.59 \\
$0: 05: 51.194$ & $+32: 56: 35.75$ & $18.075 \pm 0.020$ & $-250.5 \pm 8.7$ & -44.9 & 112.01 & -28.96 \\
$0: 07: 00.445$ & $-19: 46: 13.52$ & $18.304 \pm 0.029$ & $8.9 \pm 9.7$ & 55.0 & 66.44 & -77.44 \\
$0: 07: 57.158$ & $-06: 02: 46.37$ & $18.123 \pm 0.017$ & $-124.7 \pm 12.4$ & -27.6 & 94.85 & -66.52 \\
$0: 12: 26.890$ & $-10: 47: 54.56$ & $19.042 \pm 0.025$ & $-128.2 \pm 8.7$ & -51.0 & 91.74 & -71.27 \\
$0: 13: 00.653$ & $-20: 23: 31.26$ & $18.511 \pm 0.071$ & $-40.4 \pm 11.3$ & 0.1 & 68.37 & -78.93 \\
$0: 15: 58.669$ & $+33: 48: 40.41$ & $17.494 \pm 0.018$ & $8.2 \pm 11.0$ & 210.3 & 114.56 & -28.48 \\
$0: 17: 22.694$ & $+32: 33: 54.26$ & $19.592 \pm 0.019$ & $46.9 \pm 11.3$ & 246.1 & 114.67 & -29.76 \\
\hline
\end{tabular}

Note. - (This table is available in its entirety in machine-readable and Virtual Observatory forms in the online journal. A portion is shown here for guidance regarding its form and content.)

Piffl, T., Williams, M., \& Steinmetz, M. 2011, A\&A, 535, A70

Portegies Zwart, S. F. 2000, ApJ, 544, 437

Portegies Zwart, S. F., Baumgardt, H., McMillan, S. L. W., et al. 2006, ApJ, 641, 319

Poveda, A., Ruiz, J., \& Allen, C. 1967, Bol. Obs Tonantzintla Tacubaya, 4, 860

Przybilla, N., Nieva, M. F., Heber, U., \& Butler, K. 2008a, ApJ, 684, L103

Przybilla, N., Nieva, M. F., Heber, U., et al. 2008b, A\&A, 480, L37

Przybilla, N., Nieva, M. F., Tillich, A., et al. 2008c, A\&A, 488, L51

Przybilla, N., Tillich, A., Heber, U., \& Scholz, R.-D. 2010, ApJ, 718,37

Reid, M. J., Menten, K. M., Zheng, X. W., et al. 2009, ApJ, 700, 137

Sakai, S., Madore, B. F., \& Freedman, W. L. 1997, ApJ, 480, 589

Sari, R., Kobayashi, S., \& Rossi, E. M. 2010, ApJ, 708, 605

Schaller, G., Schaerer, D., Meynet, G., \& Maeder, A. 1992 A\&AS, 96, 269

Schmidt, G. D., Weymann, R. J., \& Foltz, C. B. 1989, PASP, 101,713

Schönrich, R., Binney, J., \& Dehnen, W. 2010, MNRAS, 403, 1829

\section{APPENDIX}

\section{DATA TABLE}

Table 2 presents the sample of 955 late B-type stars in the HVS Survey studied here. For completeness, we provide both the 375 newly observed objects and the 580 previously reported objects. Columns include J2000 coordinates, SDSS $g$-band apparent magnitude, our heliocentric velocity, velocity in the Galactic rest frame (Equation 1), and Galactic longitude and latitude.
Sesana, A., Haardt, F., \& Madau, P. 2006, ApJ, 651, 392

-. 2007a, ApJ, 660, 546

- 2007b, MNRAS, 379, L45

2008, ApJ, 686, 432 Sherwin, B. D., Loeb, A., \& O'Leary, R. M. 2008, MNRAS, 386 1179

Smith, M. C., Ruchti, G. R., Helmi, A., et al. 2007, MNRAS, 379, 755

Svensson, K. M., Church, R. P., \& Davies, M. B. 2008, MNRAS, 383, L15

Tauris, T. M. \& Takens, R. J. 1998, A\&A, 330, 1047

Tillich, A., Przybilla, N., Scholz, R., \& Heber, U. 2009, A\&A, 507, L37

Vilardell, F., Ribas, I., Jordi, C., Fitzpatrick, E. L., \& Guinan,

E. F. 2010, A\&A, 509, A70

Wu, X., Famaey, B., Gentile, G., Perets, H., \& Zhao, H. 2008, MNRAS, 386, 2199

Yanny, B., Rockosi, C., Newberg, H. J., et al. 2009, AJ, 137, 4377

Yu, Q. \& Madau, P. 2007, MNRAS, 379, 1293

Yu, Q. \& Tremaine, S. 2003, ApJ, 599, 1129

Zhang, F., Lu, Y., \& Yu, Q. 2010, ApJ, 722, 1744 